This item was submitted to Loughborough's Research Repository by the author.

Items in Figshare are protected by copyright, with all rights reserved, unless otherwise indicated.

\title{
A randomized integral error criterion for parametric identification of dynamic models of mechanical systems
}

PLEASE CITE THE PUBLISHED VERSION

PUBLISHER

Professional Engineering Publishing / @ IMechE

VERSION

VoR (Version of Record)

LICENCE

CC BY-NC-ND 4.0

\section{REPOSITORY RECORD}

Gordon, T.J., and Matt C. Best. 2009. "A Randomized Integral Error Criterion for Parametric Identification of Dynamic Models of Mechanical Systems". figshare. https://hdl.handle.net/2134/5112. 
This item was submitted to Loughborough's Institutional Repository (https://dspace.lboro.ac.uk/) by the author and is made available under the following Creative Commons Licence conditions.

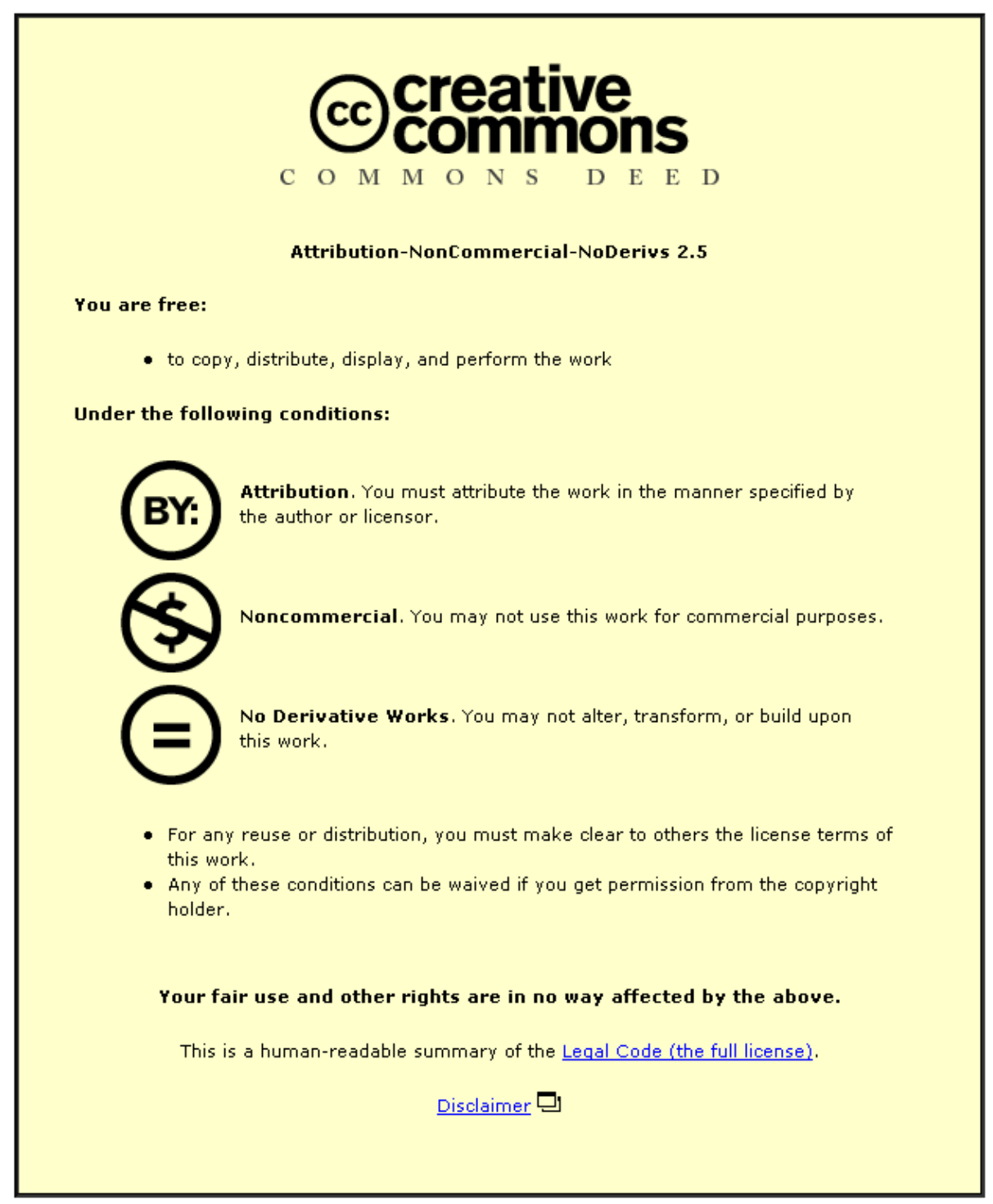

For the full text of this licence, please go to: http://creativecommons.org/licenses/by-nc-nd/2.5/ 


\title{
A randomized integral error criterion for parametric identification of dynamic models of mechanical systems
}

M C Best* and T J Gordon

Department of Aeronautical and Automotive Engineering, Loughborough University, Leicestershire, UK

\begin{abstract}
This paper proposes a new approach to the identification of reduced order models for complex mechanical vibration systems. Parametric identification is commonly conducted by the regression of time-series data, but when this includes significant unmodelled modes, the error process has a high variance and autocorrelation. In such cases, optimization using least-squares methods can lead to excessive parameter bias. The proposed method takes advantage of the inherent boundedness of mechanical vibrations to design a new regression set with dramatically reduced error variance.

The principle is first demonstrated using a simple two-mass simulation model, and from this a practicable approach is derived. Extensive investigation of the new randomized integral error criterion method is then conducted using the example of identification of a quarter-car suspension system. Simulation results are contrasted with those from comparable direct least-squares identifications. Several forms of the identification equations and error sources are used, and in all cases the new method has clear advantages, both in accuracy and consistency of the resulting identification model.
\end{abstract}

Keywords: system identification, parametric identification, mechanical systems, vehicle suspension

\section{NOTATION}

$B_{\mathrm{m}}$

$E_{1}$

$E_{2}$

$f_{\text {max }}$

$F_{\mathrm{d} i}$

$F_{i}$

$F_{\mathrm{s}}, F_{\mathrm{d}}, F_{\mathrm{ms}}, F_{\mathrm{t}}$

$K_{\mathrm{c}}$

$K_{\mathrm{m}}$

$K_{\mathrm{s}}$

$K_{\mathrm{t}}$ mount damping constant for source model ordinary least-squares error norm randomized integral error norm cut-off frequency applied to the simulation input process; input bandwidth damping force acting in the identification model

damper force parameters for the identification model

suspension, damping, mount spring and tyre forces respectively acting in the source model

damper compliance of the source model

linearized mount stiffness of the source model

suspension stiffness of the identification model

tyre stiffness of the source and the identification models

The MS was received on 24 July 1997 and was accepted after revision for publication on 7 October 1998.

* Corresponding author: Lecturer in Dynamics and Control, Department of Aeronautical and Automotive Engineering, Loughborough University, Leicestershire LE11 3TU, UK.
$M_{\mathrm{w}}, M_{\mathrm{b}}, M_{\mathrm{e}} \quad$ unsprung (wheel), sprung (body) and engine masses respectively of the source model

$M_{\mathrm{us}}, M_{\mathrm{s}} \quad$ unsprung and sprung masses respectively of the identification model

$p_{i} \quad$ percentage validation error for the $i$ th state variable

$R_{i} \quad$ regressors for the identification model damper map

data batch length

start time for the integration

integration interval

suspension velocity break points in the identification model damper map road velocity input process for the simulation exercise

$x_{1}, x_{2}, x_{3}, x_{4}, x_{5}, x_{6}, x_{7}$

state variables for the source and the identification models direct application of ordinary leastsquares

randomized integral error criterion 


\section{INTRODUCTION}

This paper concerns the parametric identification of mechanical system models, i.e. the process of defining an underlying mathematical model for the dynamic system of interest, and estimating free parameters to give the 'best fit' against measured experimental data. For example, in vehicle ride dynamics, the input data might be from time histories of vertical tyre contact forces, and the output variables could be the resulting displacements and accelerations recorded for motion of the vehicle body. The accuracy (and hence the usefulness) of system identification depends on a number of factors, particularly in the choices for model structure and input excitations. Another significant factor is the choice of error criterion, i.e. the objective measure of goodness of fit between the system model and the physical measurements. See, for example, references [1] and [2] for standard approaches in the identification of linear systems.

In the simplest case, the model equations can be written as follows:

$$
y(t)=f[u(t), \theta]+e(t)
$$

where $y(t)$ is a vector of measured outputs, $u(t)$ a corresponding vector of inputs (or other measured system variables), $\theta$ denotes a set of constant unknown parameters and $e(t)$ is an 'error' term. The errors essentially represent unmodelled effects in the physical dynamic system, including any higher-order vibrational modes, electrical noise and the effects of transducer crosssensitivity. Of course these error signals are not known or independently measured, but they can be estimated from equation (1) for any postulated parameter set $\theta$. Intuitively, the best choice of parameters is that which minimizes these 'unexplained' equation errors, with error criterion defined by

$$
E_{1}=\sum_{i}\left\|y\left(t_{i}\right)-f\left[u\left(t_{i}\right), \theta\right]\right\|^{2}
$$

where the summation is over the available set of data points. The corresponding estimator $\hat{\theta}$ is the mapping that provides parameters to minimize $E_{1}$. In the ideal case, where the errors actually arise as a white noise process, uncorrelated with the input variables $u(t)$, an optimal choice, namely the minimum-variance unbiased estimator, is indeed obtained in this way [1]. The required minimization can be carried out using one of many available numerical algorithms for non-linear least-squares optimization, for example the LevenbergMarquardt algorithm (see, for example, reference [3]).

If, in addition, the model equations are linear in the unknown parameters, the problem has a standard explicit and unique solution. For data collected at $n$ time instants, the model may be written in the following matrix-vector form [for notational simplicity the under- lying model (1) is taken as scalar here]

$$
\begin{aligned}
{\left[\begin{array}{c}
y\left(t_{1}\right) \\
y\left(t_{2}\right) \\
\vdots \\
y\left(t_{n}\right)
\end{array}\right]=} & {\left[\begin{array}{cccc}
a_{1}\left[u\left(t_{1}\right)\right] & a_{2}\left[u\left(t_{1}\right)\right] & \cdots & a_{p}\left[u\left(t_{1}\right)\right] \\
a_{1}\left[u\left(t_{2}\right)\right] & a_{2}\left[u\left(t_{2}\right)\right] & \cdots & a_{p}\left[u\left(t_{2}\right)\right] \\
\vdots & \vdots & \vdots & \vdots \\
a_{1}\left[u\left(t_{n}\right)\right] & a_{2}\left[u\left(t_{n}\right)\right] & \cdots & a_{p}\left[u\left(t_{n}\right)\right]
\end{array}\right]\left[\begin{array}{c}
\theta_{1} \\
\theta_{2} \\
\vdots \\
\theta_{p}
\end{array}\right] } \\
& +\left[\begin{array}{c}
e\left(t_{1}\right) \\
e\left(t_{2}\right) \\
\vdots \\
e\left(t_{n}\right)
\end{array}\right]
\end{aligned}
$$

or more simply

$$
y=A \theta+e
$$

This leads to the well-known least-squares estimate [1]

$$
\hat{\theta}=\left(A^{\mathrm{T}} A\right)^{-1} A^{\mathrm{T}} y
$$

In this paper, this direct approach of minimizing model equation error will be referred to as the direct ordinary least-squares (DOLS) method. Perhaps the most severe limitation of this basic approach is the assumption that the error signal $e(t)$ arises as an idealized white noise process, uncorrelated with the elements of the regressor matrix $A$. If this is not the case, the estimator may suffer from systematic bias and/or excessive variance. For example, in vehicle ride dynamics, errors arising from unmodelled mechanical vibrations have associated resonance frequencies, and $e(t)$ is clearly not white noise. Also, unmodelled non-linearities are likely to induce correlations between $e(t)$ and the regressor matrix, while errors in the regressors themselves induce bias $[\mathbf{1}, \mathbf{2}]$.

Techniques such as the generalized least-squares method and the maximum-likelihood estimation method $[1,2]$ may be used to overcome some of these limitations but require explicit knowledge of the statistics or probability models underlying the error processes. On the other hand, any mechanical system is subject to the fundamental principles of energy and momentum conversion, and this applies as much to the unmodelled degrees of freedom as to those that are modelled. If the errors in a simplified model are constrained by such principles, it may be possible to apply suitable transformations to reduce the relative size of the error terms and hence to reduce the variance in the parameter estimators.

In this paper, attention is directed to the elementary principle of impulse-momentum, whereby the impulse of a force is equal to the net momentum transferred in the corresponding degree of freedom; this leads to a randomized integral error criterion (RIEC) identification procedure. A quarter-vehicle automotive suspension model will be used to investigate the usefulness of this new technique. In Section 2, the general concept is introduced and the error properties are considered in the context of a two-mass model. Relevant quarter-car models 
are defined in Section 3. Section 4 details the identification procedures, and in Section 5 the performance of the RIEC method is compared with that of the simpler DOLS identification, for a reference case. The robustness of these two procedures is then investigated in Section 6, particularly in respect of input signal requirements.

\section{RANDOMIZED INTEGRAL ERROR CRITERION}

Figure 1 represents a very simple example of a system where equation errors arise via an unmodelled vibrational mode. The left-hand diagram represents a 'physical' two-degree-of-freedom (2 DOF) system where a small mass $m_{2}$ is flexibly coupled to a larger mass $m_{1}$ which moves vertically under the action of some force $F(t)$. In general, $F(t)$ might arise via interactions with other parts of a dynamic system, so that it takes the form $F(t)=F[x(t), \theta]$. Then the dynamic equation of vertical motion can be used to help identify the unknown parameters $\theta$. The right-hand diagram represents a simplified one-degree-of-freedom (1 DOF) model that might be used in identification. The motion of the lumped mass is inferred from the motion sensor (accelerometer) attached to the lower mass in the 2 DOF system. Owing to the simplified representation, this induces an equation error $\Delta F(t)$, and the motion of the simplified system is given by

$$
\left(m_{1}+m_{2}\right) \ddot{z}=F(t)+\Delta F(t)
$$

It is easily shown that $\Delta F(t)$ is an 'inertial' force associated with the relative deflection $d(t)$ of $m_{2}$ relative to $m_{1}$ in the 2 DOF model:

$$
\Delta F(t)=m_{2} \ddot{d}
$$

This is valid however the internal forces $F_{\text {int }}(t)$ are generated-perhaps via non-linear spring and damping elements which may be subject to hysteresis and dry friction.

Although at any instant the magnitude of the equation error can be large compared with the other terms, it may be constrained in its cumulative effect. Integrating equa-

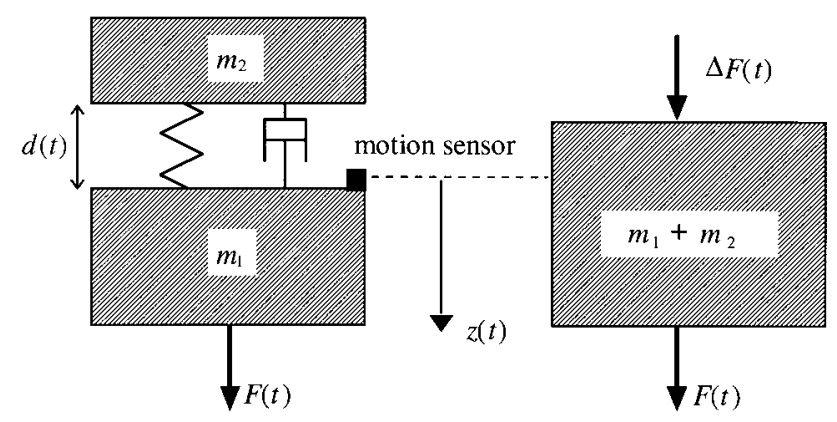

Fig. 1 Unmodelled mode resulting in equation error tion (5) over a time interval $\left(t_{1}, t_{2}\right)$ gives

$$
\left(m_{1}+m_{2}\right)\left[\dot{z}\left(t_{2}\right)-\dot{z}\left(t_{1}\right)\right]=\int_{t_{1}}^{t_{2}} F(t) \mathrm{d} t+\int_{t_{1}}^{t_{2}} \Delta F(t) \mathrm{d} t
$$

This is the impulse-momentum equation for the dynamics of the $1 \mathrm{DOF}$ model. The integral of the error is associated with a net momentum transfer, which remains bounded even over long time intervals, provided that the relative velocity of the unmodelled degree of freedom is bounded by some value $v_{\max }$ :

$$
\int_{t_{1}}^{t_{2}} \Delta F(t) \mathrm{d} t=m_{2}\left[\dot{d}\left(t_{2}\right)-\dot{d}\left(t_{1}\right)\right] \leqslant 2 m_{2} v_{\max }
$$

Thus, if the time interval is chosen so that relatively large momentum changes occur in the lumped mass $m_{1}+m_{2}$, the effect of the integration should be to reduce the relative magnitude of the equation error. (In principle, although a second integration might be performed to give error bounds based on peak deflections, this will not be considered in this paper.)

The possible effectiveness of the time integration is illustrated in Fig. 2. Figure 2a shows an 'arbitrary' error signal (a sample of Gaussian white noise) while Fig. $2 b$ is a simulated time history for $\Delta F(t)$ in the above $1 \mathrm{DOF}$ system. $F(t)$ was applied to the $2 \mathrm{DOF}$ model as Gaussian white noise, and a linear spring damper combination was chosen to act between $m_{1}$ and $m_{2}$, using the following parameters:

$$
\begin{aligned}
& m_{1}=10 \mathrm{~kg}, \quad m_{2}=1 \mathrm{~kg} \\
& \omega_{n}=100 \mathrm{rad} / \mathrm{s}, \quad \zeta=0.5
\end{aligned}
$$

The magnitude of $F(t)$ was chosen such that the two error signals $e(t)$ in Figs $2 \mathrm{a}$ and $\mathrm{b}$ have the same r.m.s. values.

The two error signals look broadly similar in the plots, but the effect of integration is markedly different; see Figs $2 \mathrm{c}$ and $\mathrm{d}$, where the corresponding integral error

$$
I(t)=\int_{0}^{t} e(t) \mathrm{d} t
$$

is shown. The arbitrary white noise signal leads to drift, as a typical 'random walk' process in Fig. 2c, while the effective constraint on maximum velocity strongly bounds the integral error in Fig. 2 d.

The way in which these considerations can be turned into a practical technique for system identification is now considered. The integration used in equation (9) will provide an alternative set of equations for error minimization; for example equation (7) gives rise to the set

$$
\begin{array}{r}
\left(m_{1}+m_{2}\right)\left[\dot{z}\left(t_{i}\right)-\dot{z}(0)\right]=\int_{0}^{t_{i}} F[x(t), \theta] \mathrm{d} t+I\left(t_{i}\right) \\
(i=1,2, \ldots, N)
\end{array}
$$


(a) Before integration

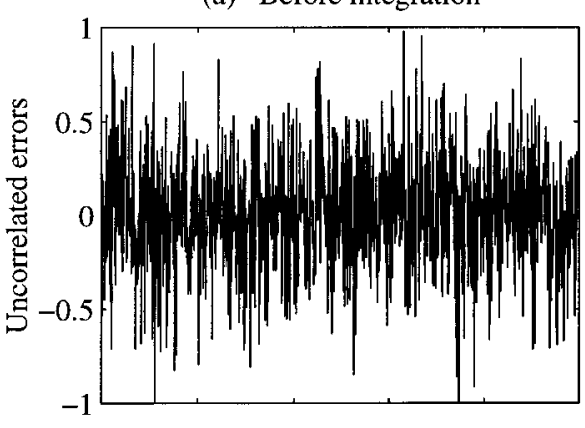

(b)

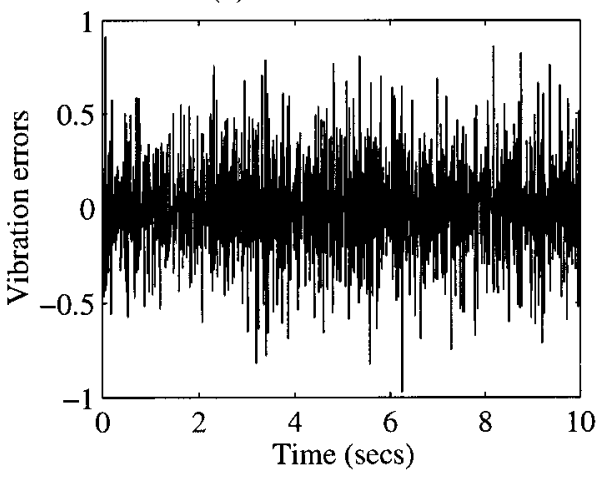

(c) After integration

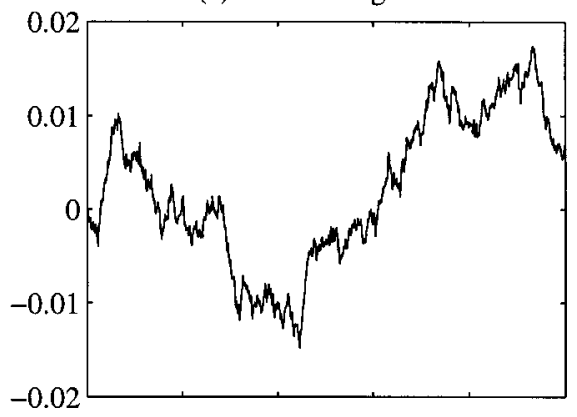

(d)

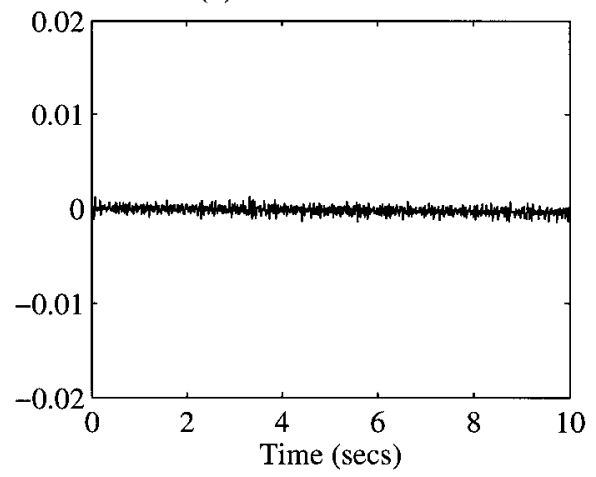

Fig. 2 Comparison of uncorrelated and mechanical equation errors

This is not particularly useful as it stands, because of the following limitations:

1. Integration over successively longer time intervals makes the transformed model equations unduly sensitive to steady state and very-low-frequency contributions to $e(t)$, such as amplifier drift and transducer offset.

2. There is a systematic weighting, or over-sampling, associated with data at the start of the test, i.e. near $t=0$, while there is no a priori reason to prefer these data to those obtained at later times.

3. Related to item 1, the integral errors, obtained from variable overlapping time intervals, are statistically correlated in a way that is impossible to characterize without detailed knowledge of the spectral properties of the underlying error processes.

To avoid problem 1 it is simple to apply upper bounds on the integration time $T$, while item 2 motivates selection of a lower integration limit $t_{0}$. If $t_{0}$ is then selected by random sampling, the correlations noted in item 3 are also avoided. The transfer function for this modified integration process can be seen as the difference between two delayed pure integrators:

$$
I(t)=\int_{t_{0}}^{t_{0}+T} e(t) \mathrm{d} t
$$

Therefore

$$
I(s)=H(s) e(s)
$$

where

$$
H(s)=\frac{\mathrm{e}^{-s\left(t_{0}+T\right)}}{s}-\frac{\mathrm{e}^{-s t_{0}}}{s}=\frac{1-\mathrm{e}^{-s T}}{s} \mathrm{e}^{-s t_{0}}
$$

The corresponding frequency response gain is then easily found to be

$$
|H(\mathrm{j} \omega)|=\frac{2 \sin (\omega T / 2)}{\omega} \equiv T \operatorname{sinc}\left(\frac{\omega T}{2}\right)
$$

Choosing for example $T=0.1 \mathrm{~s}$, this is plotted as the solid curve in Fig. 3. Unfortunately the operation is 'tuned' to give zero gain (drop-out) at discrete frequencies, here at multiples of $10 \mathrm{~Hz}$. Rejecting data at certain discrete frequencies in this way cannot be satisfactory in general. Figure 3 also shows the effect of averaging over two distinct time intervals: $T=0.1 \mathrm{~s}$ and $T=0.33 \mathrm{~s}$, with clearly improved results. Averaging over a continuous range of sample times, from $T=0.1 \mathrm{~s}$ to $T=1.0 \mathrm{~s}$ gives the third smooth curve which, although biased towards low frequencies, does not suffer from discrete frequency drop-out. The same effect is achieved for the expected frequency response if the operator incorporates random sampling of $T$ from the corresponding uniform probability distribution

$$
T \sim U(\alpha, \beta)
$$

with $\alpha=0.1$ and $\beta=1.0$. Taken together with a random choice of the initial time

$$
t_{0} \sim U\left(0, t_{\max }\right)
$$




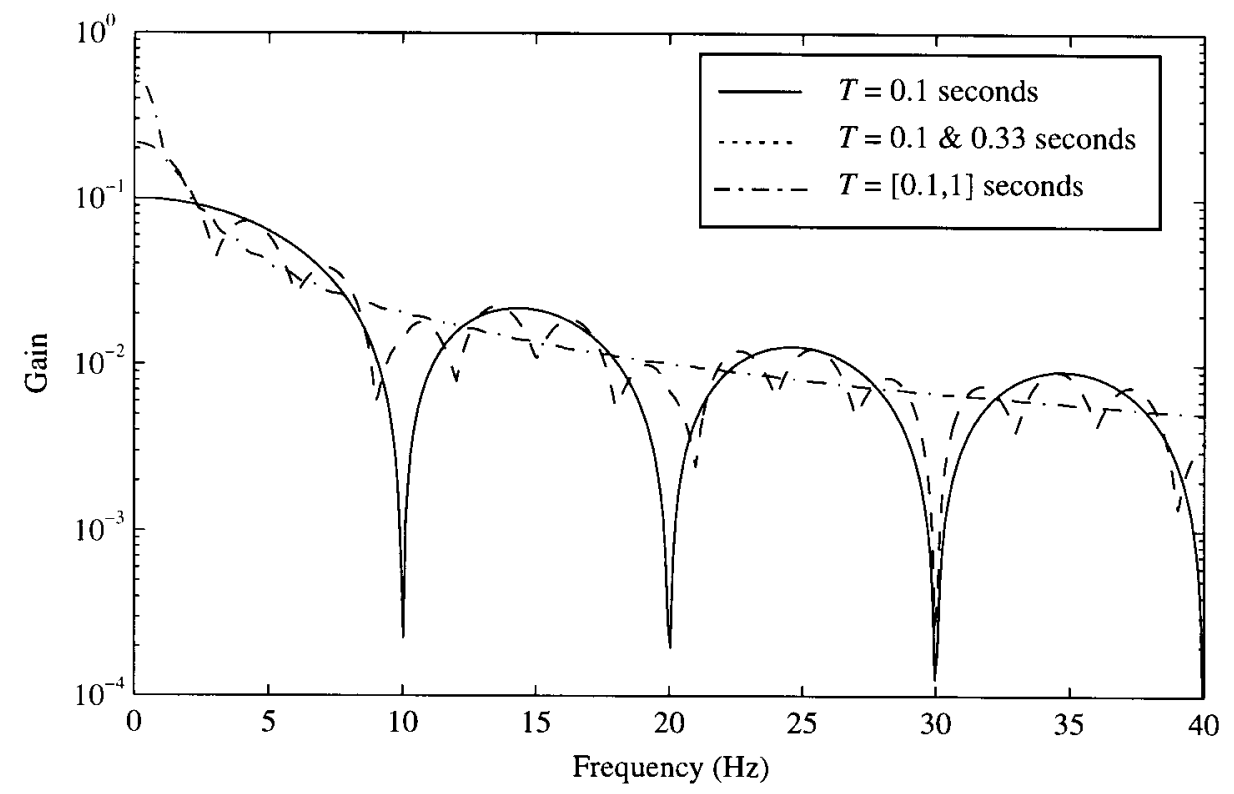

Fig. 3 Frequency response gain of the integration process

there is a need to reject the chosen values for $T$ and $t_{0}$ whenever $t_{0}+T>t_{\max }$. Choice of the free parameters $\alpha$ and $\beta$ should depend on the approximate bandwidth of the model being identified, and this point is briefly taken up in Section 4.

In view of the proposed use of random sampling of data from within a given batch, item 3 above is actually addressed automatically, and in fact the statistical properties of the equation errors will be stated below explicitly, simply on the grounds of symmetry.

In the general case, typical model equations can be written

$$
M \ddot{q}=F(q, \dot{q}, t ; \theta)
$$

where $q$ is a vector of generalized displacements, $M$ is the mass matrix and $F$ represents generalized forces. In the integral form the error criterion is

$$
\begin{aligned}
E_{2}=\sum \| M\left[\dot{q}\left(t_{0}+T\right)-\dot{q}\left(t_{0}\right)\right] \\
\\
\quad-\int_{t_{0}}^{t_{0}+T} F[q(t), \dot{q}(t), t ; \theta] \mathrm{d} t \|^{2}
\end{aligned}
$$

the summation being over the randomly selected time intervals. This, formally, is the RIEC.

Returning to error statistics, and reverting to the notation $e$ for equation error (now in the integral form) it is clear that for a randomly selected time interval there corresponds a constant expected value for the mean square of equation error:

$$
E\left\{e_{i}^{2}\right\}=\sigma^{2}
$$

while for any two different time intervals there is a constant cross-correlation which can be written

$$
E\left\{e_{i} e_{j}\right\}=\sigma^{2} \varepsilon
$$

and typically $\varepsilon \ll 1$ provided that $T \ll t_{\max }$. Thus, even though the error processes are completely unknown, the covariance matrix of equation errors can be given as

$$
E\left[e^{\mathrm{T}} e\right]=\sigma^{2}\left[\begin{array}{ccccc}
1 & \varepsilon & \varepsilon & \cdots & \varepsilon \\
\varepsilon & 1 & \varepsilon & \cdots & \varepsilon \\
\varepsilon & \varepsilon & 1 & \cdots & \varepsilon \\
\cdots & \cdots & \cdots & \cdots & \cdots \\
\varepsilon & \varepsilon & \varepsilon & \cdots & 1
\end{array}\right]
$$

From this, it is possible to apply the generalized leastsquares method to the minimization of $E_{2}$, and it has been shown that the RIEC optimal solution is achieved by applying the standard linear least-squares method to the integral equations, provided that a sufficiently large number of samples are employed, and provided that the regressors have zero mean [4]. Both of these conditions are met in the following simulation study.

\section{SIMULATION MODELS}

Identification of a simple model for a vehicle ride provides a suitable test case for the new method. The wellknown quarter-vehicle model, considered extensively as a platform for suspension control in the literature, is used. Two variants are employed, as shown in Fig. 4. State variables are clearly shown as relative displacements $x_{1}, x_{2}$ and $x_{5}$ and velocities (bold arrows).

The model in Fig. 4a, used to provide source data for the identification, is termed the source model. It incorporates a nominal representation of several physical characteristics that are typically unmodelled, combining a high level of non-linearity with additional degrees of freedom 


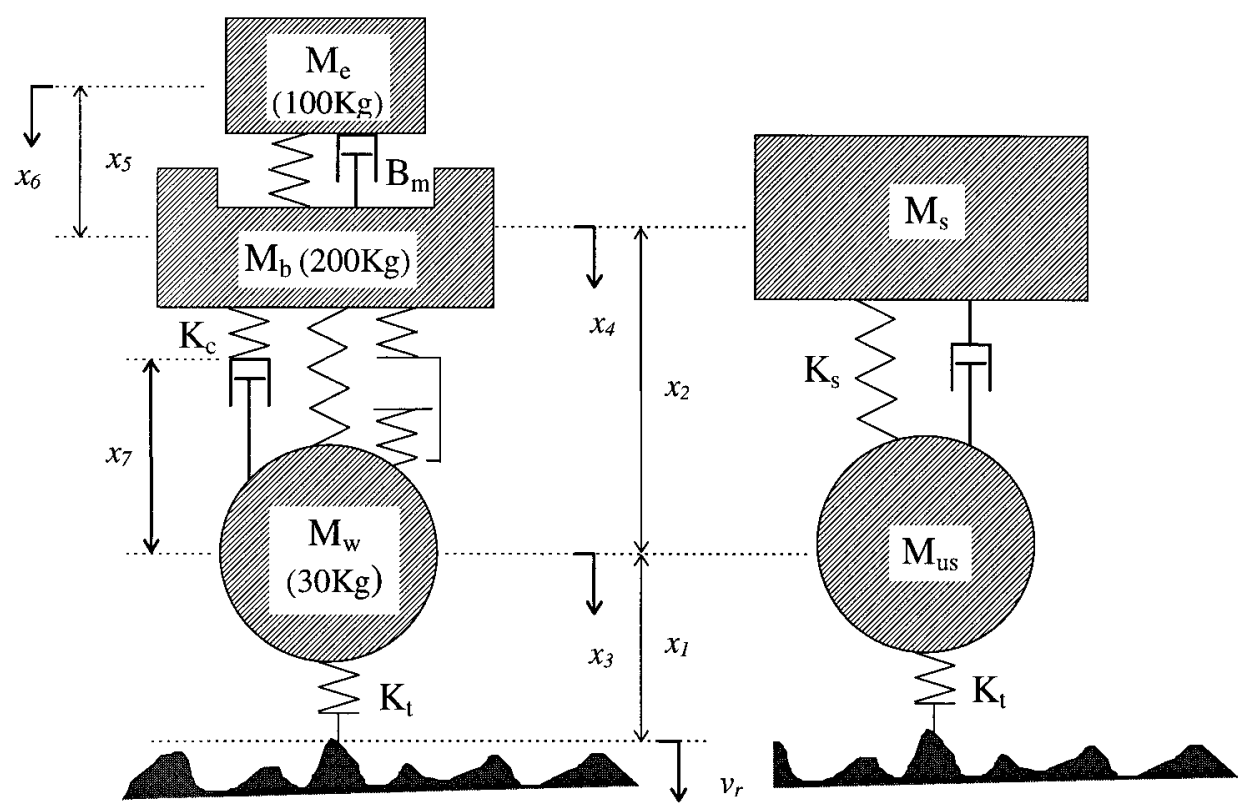

(a) Source model

(b) Identification model

Fig. 4 Models for source data and identification

in vertical engine vibrations and compliance in suspension damping. The dynamic equations of motion are

$$
\begin{aligned}
& \dot{x}_{1}=v_{\mathrm{r}}-x_{3} \\
& \dot{x}_{2}=x_{3}-x_{4} \\
& \dot{x}_{3}=\frac{1}{M_{\mathrm{w}}}\left(F_{\mathrm{t}}-F_{\mathrm{s}}-F_{\mathrm{d}}\right) \\
& \dot{x}_{4}=\frac{1}{M_{\mathrm{b}}}\left[F_{\mathrm{s}}+F_{\mathrm{d}}-F_{\mathrm{ms}}-B_{\mathrm{m}}\left(x_{4}-x_{6}\right)\right] \\
& \dot{x}_{5}=x_{4}-x_{6} \\
& \dot{x}_{6}=\frac{1}{M_{\mathrm{e}}}\left[F_{\mathrm{ms}}+B_{\mathrm{m}}\left(x_{4}-x_{6}\right)\right] \\
& \dot{x}_{7}=K_{\mathrm{c}}\left(\frac{\mathrm{d} F_{\mathrm{d}}}{\mathrm{d} x_{7}}\right)^{-1}\left(x_{3}-x_{4}-x_{7}\right)
\end{aligned}
$$

The tyre force $F_{\mathrm{t}}$ is modelled as a linear spring with a threshold to accommodate lifting of the tyre contact patch from the simulated road surface:

$$
F_{\mathrm{t}}= \begin{cases}K_{\mathrm{t}} x_{1}, & x_{1} \leqslant \frac{W_{\mathrm{t}}}{K_{\mathrm{t}}} \\ W_{\mathrm{t}}, & x_{1}>\frac{W_{\mathrm{t}}}{K_{\mathrm{t}}}\end{cases}
$$

where $\quad W_{\mathrm{t}}=\left(M_{\mathrm{e}}+M_{\mathrm{b}}+M_{\mathrm{w}}\right) g$ and $K_{\mathrm{t}}=180 \mathrm{~N} / \mathrm{mm}$. Note that the threshold force is non-zero, as the dynamic state variables are taken as zero at the static load point.

The suspension forces, $F_{\mathrm{d}}$ and $F_{\mathrm{s}}$ and the mount spring force $F_{\mathrm{ms}}$ are modelled with piecewise linear and cubic spline functions, illustrated in Fig. 5. For small excitations the source model can be linearized, allowing a modal analysis. Approximating the suspension damping rate at $1.5 \mathrm{kN} \mathrm{s} / \mathrm{m}$ and using the first-stage suspension and mount spring stiffnesses, $20 \mathrm{kN} / \mathrm{m}$ and $400 \mathrm{kN} / \mathrm{m}$ respectively, the resulting eigenstructure is summarized in Table 1.

The resonance frequencies are typical of suspension system modes, commonly referred to as 'wheel hop' at $13 \mathrm{~Hz}$, and 'body bounce' at $1 \mathrm{~Hz}$, but here the system also includes a pole due to the damper compliance freedom, and a third dynamic mode due to the engine motion. Note that the engine and wheel-hop modes both appear at approximately $13 \mathrm{~Hz}$; this frequency matching is typical of physical suspension systems, and the eigenvectors for these two modes show that the wheel vibrations are strongly coupled with the motion of the engine relative to the body. The model thus provides a pathological case for identification, as higher-frequency excitations required to characterize wheel hop will also maximize the disturbing influence of the engine mass. The very-low-magnitude wheel velocity component in the body-bounce mode indicates that it would not be sensible to identify the model from low-frequency excitations alone.

The two-mass identification model of Fig. $4 \mathrm{~b}$ employs only four state variables and has entirely linear components except in the damper characteristic. The equations of motion are

$$
\begin{aligned}
& \dot{x}_{1}=v_{\mathrm{r}}-x_{3} \\
& \dot{x}_{2}=x_{3}-x_{4} \\
& \dot{x}_{3}=\frac{1}{M_{\mathrm{us}}}\left(K_{\mathrm{t}} x_{1}-K_{\mathrm{s}} x_{2}-F_{\mathrm{d} i}\right) \\
& \dot{x}_{4}=\frac{1}{M_{\mathrm{s}}}\left(K_{\mathrm{s}} x_{2}+F_{\mathrm{d} i}\right)
\end{aligned}
$$


(a)

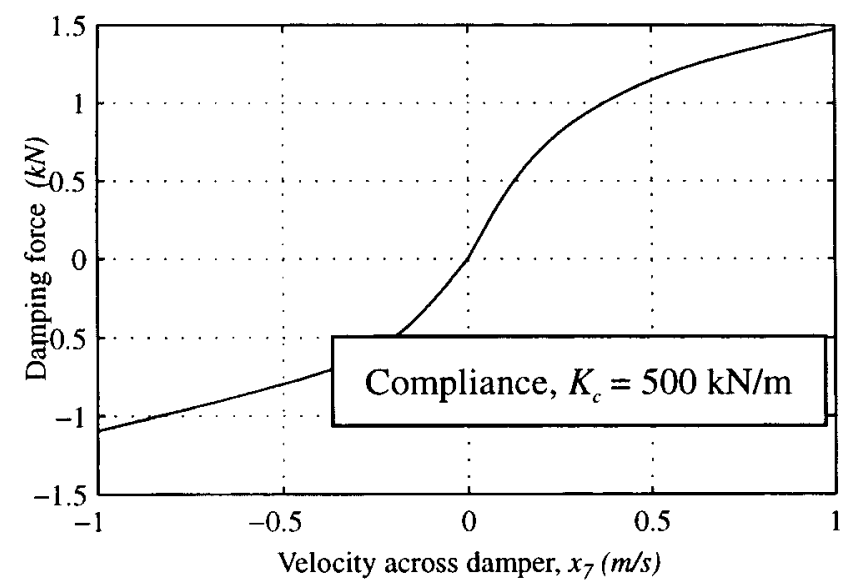

(c)

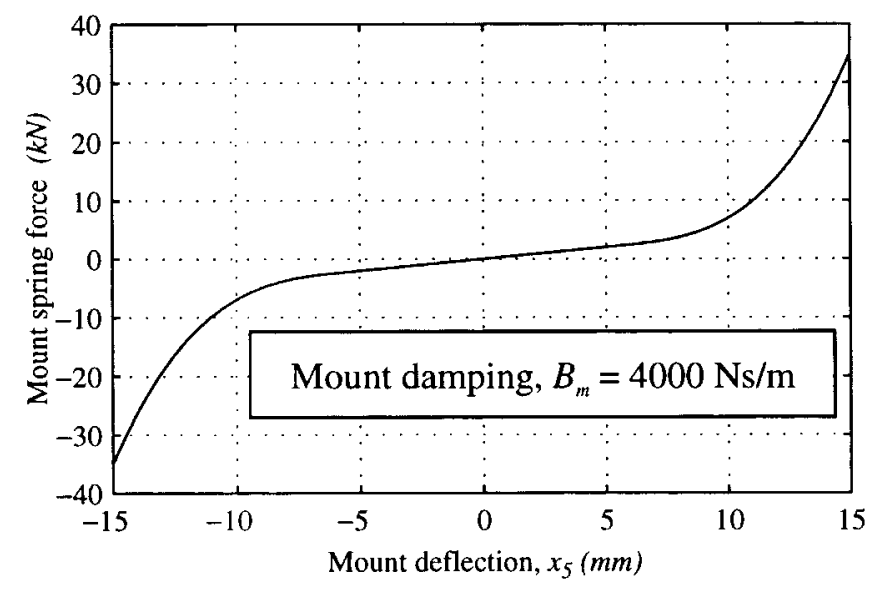

(b)

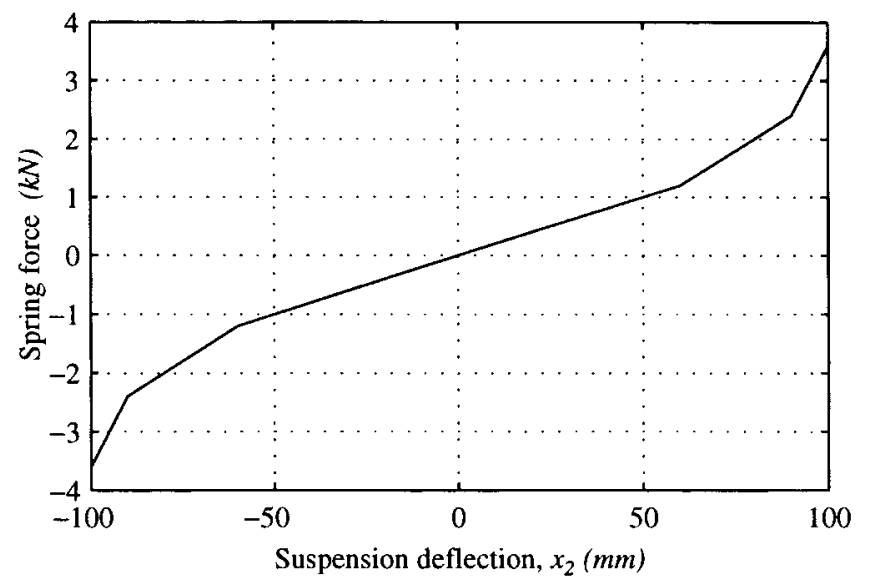

Fig. 5 Non-linear characteristics of the source model: (a) damper, (b) spring and bumpstops, (c) engine mount

Table 1 The eigenstructure of the linearized source model

\begin{tabular}{|c|c|c|c|c|c|}
\hline \multirow[b]{2}{*}{ Eigenvalues } & \multirow{2}{*}{$\begin{array}{l}\text { Frequency } \\
(\mathrm{Hz})\end{array}$} & \multirow[b]{2}{*}{$\xi$} & \multicolumn{3}{|c|}{ Eigenvector velocity components } \\
\hline & & & Wheel & Body & Engine-body \\
\hline-266 & - & - & 1.00 & -0.17 & 0.19 \\
\hline$-35.15 \pm 75.76 \mathrm{i}$ & 13.29 & 0.42 & 1.00 & $-0.21 \mp 0.29 \mathrm{i}$ & $0.49 \pm 0.67$ \\
\hline$-26.21 \pm 78.75 \mathrm{i}$ & 13.21 & 0.32 & 1.00 & $0.07 \mp 0.19 \mathrm{i}$ & $-0.29 \pm 0.41$ \\
\hline$-2.08 \pm 7.63 \mathrm{i}$ & 1.26 & 0.26 & $0.09 \pm 0.05$ & 1.00 & $0.01 \pm 0.01$ \\
\hline
\end{tabular}

where $F_{\mathrm{d} i}$ takes a simple piecewise linear form, as illustrated in Fig. 6a. For identification, this can be written as a linear function in four force values $F_{i}$ :

$$
F_{\mathrm{d} i}=\sum_{i=1}^{4} F_{i} R_{i}\left(v_{i}, x_{3}-x_{4}\right)
$$

where the $R_{i}$ are discontinuous linear 'roof' functions of suspension velocity $x_{3}-x_{4}$ bounded according to preI04497 ( ) IMechE 1999 determined velocity break points $v_{i}$; the $R_{i}$ are illustrated in Fig. 6b.

\section{IDENTIFICATION PROCEDURE}

The simulated identifications are carried out using source data simulated from a Gaussian white noise input pro-

Proc Instn Mech Engrs Vol 213 Part I 

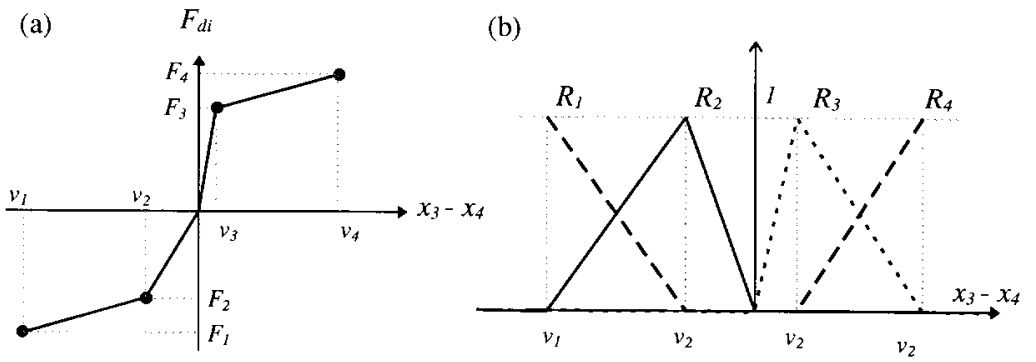

Fig. 6 (a) Piecewise linear suspension damper model and (b) regression function used for identification

cess $v_{\mathrm{r}}$, chosen with sufficiently high bandwidth to excite all modes. The amplitude of $v_{\mathrm{r}}$ was chosen to excite all non-linearities of the source model, while ensuring that the 'lifting' tyre remained in contact with the simulated road surface for 99 per cent of the test duration. Details of this and all other parameters required for the identification process are summarized in Table 2.

Using values for the state variables $x_{1}, x_{2}, x_{3}$ and $x_{4}$, their derivatives and the tyre force $F_{\mathrm{t}}$ from the source model simulation, parameter estimates are made according to the structure of the identification model. From equations (21) and (22), a series of regression equations can be formulated according to both DOLS and RIEC criteria; these are given below. For each regression the estimated parameters, denoted with a circumflex ${ }^{\wedge}$, are obtained by least-squares solution of the matrix formed from a large number of samples, $N$, according to the structure of equation (3).

The DOLS formulation is

$$
\begin{aligned}
& F_{\mathrm{t}}=\hat{K}_{\mathrm{t}} x_{1} \\
& F_{\mathrm{t}}=\hat{M}_{\mathrm{us}} \dot{x}_{3}+\hat{M}_{\mathrm{s}} \dot{x}_{4} \\
& F_{\mathrm{t}}-M_{\mathrm{us}} \dot{x}_{3}=\hat{K}_{\mathrm{s}} x_{2}+\sum_{i=1}^{4} \hat{F}_{i} R_{i}\left(x_{3}-x_{4}\right) \\
& M_{\mathrm{s}} \dot{x}_{4}=\hat{K}_{\mathrm{s}} x_{2}+\sum_{i=1}^{4} \hat{F}_{i} R_{i}\left(x_{3}-x_{4}\right)
\end{aligned}
$$

The RIEC formulation is

$$
\begin{aligned}
& \int_{t_{0}}^{t_{0}+T} F_{\mathrm{t}}(\tau) \mathrm{d} \tau=\hat{K}_{\mathrm{t}} \int_{t_{0}}^{t_{0}+T} x_{1}(\tau) \mathrm{d} \tau \\
& \int_{t_{0}}^{t_{0}+T} F_{\mathrm{t}}(\tau) \mathrm{d} \tau=\hat{M}_{\text {us }} \Delta x_{3}+\hat{M}_{\mathrm{s}} \Delta x_{4}
\end{aligned}
$$

\begin{tabular}{|c|c|}
\hline Vertical velocity input process, $v_{\mathrm{r}}$ & $\begin{array}{l}\text { Gaussian white noise, } \\
0-30 \mathrm{~Hz}, \\
\text { r.m.s. } v_{\mathrm{r}}=0.55 \mathrm{~m} / \mathrm{s}\end{array}$ \\
\hline Test duration & $40 \mathrm{~s}$ \\
\hline Sampling rate for input and measurements & $200 \mathrm{~Hz}$ \\
\hline Damper map velocity break points, $v_{i}$ & $\left\{\begin{array}{llll}-0.8 & -0.3 & 0.3 & 0.8\end{array}\right\}$ \\
\hline For DOLS, $N_{\mathrm{s}}$ & 8000 (all available data) \\
\hline For RIEC, $N_{\mathrm{s}}$ & 8000 \\
\hline For RIEC, $\alpha$ & $0.05 \mathrm{~s}$ \\
\hline For RIEC, $\beta$ & $0.5 \mathrm{~s}$ \\
\hline
\end{tabular}

Table 2 Parameters defining the identification process

Proc Instn Mech Engrs Vol 213 Part I

$$
\begin{aligned}
& \int_{t_{0}}^{t_{0}+T} F_{\mathrm{t}}(\tau) \mathrm{d} \tau-M_{\mathrm{us}} \Delta x_{3} \\
&=\hat{K}_{\mathrm{s}} \int_{t_{0}}^{t_{0}+T} x_{2}(\tau) \mathrm{d} \tau \\
&+\sum_{i=1}^{4} \hat{F}_{i} \int_{t_{0}}^{t_{0}+T} R_{i}\left[x_{3}(\tau)-x_{4}(\tau)\right] \mathrm{d} \tau \\
& M_{\mathrm{s}} \Delta x_{4}= \hat{K}_{\mathrm{s}} \int_{t_{0}}^{t_{0}+T} x_{2}(\tau) \mathrm{d} \tau \\
&+\sum_{i=1}^{4} \hat{F}_{i} \int_{t_{0}}^{t_{0}+T} R_{i}\left[x_{3}(\tau)-x_{4}(\tau)\right] \mathrm{d} \tau
\end{aligned}
$$

Here $\Delta x \equiv x\left(t_{0}+T\right)-x\left(t_{0}\right)$, and $t_{0}$ and $T$ are generated for each sample according to the uniform distribution of equations (14) and (15):

$$
T \sim U(\alpha, \beta), \quad t_{0} \sim U\left(0, t_{\max }\right)
$$

The identification can be conducted in three stages. In either formulation, the first two regression equations provide estimates of the tyre spring rate and system masses; the third stage estimation of suspension force parameters can then be conducted using either equation (23) or equation (24). These consider force (or momentum) acting on the wheel or body respectively, employing one of the previously identified masses as a known value.

Clearly it is possible to identify all the parameters simultaneously, either by some aggregation of the regression equations or by reformulation as a matrix equation; indeed a least-squares matrix solution has been considered in reference [5]. The proposed sequential scheme is preferred, however, for two reasons. Firstly, regression separation allows a degree of differentiation between the sources of error, and hence their relative influence on parameter bias; for example, the mass parameters are estimated independently from internal suspension force non-linearities. Secondly, the redundancy in equations (23) and (24) allows a check on the consistency of the identification process.

Finally, the choices for $\alpha$ and $\beta$ in Table 2 deserve some comment. The range $0.05-0.5 \mathrm{~s}$ for the RIEC integration interval has been made to ensure high-magnitude regressors and hence to provide good matrix conditioning for the estimation of all parameters. For a sinusoid 
$x(t)$, the average absolute change

$$
|\Delta x(t)|=|\sin [2 \pi f(t+T)]-\sin (2 \pi f t)|
$$

is maximized by choosing $T=1 /(2 f)$. Thus the limits set in the integration lengths will maximize regressors over a range between nominal wheel-hop and body-bounce frequencies, $f=1 \mathrm{~Hz}$ and $f=10 \mathrm{~Hz}$. Although this assignment appears ad hoc, it has been shown in reference [4] that, within certain limits, the identified parameters are relatively insensitive to $\alpha$ and $\beta$. The main effect of changing integration intervals is on matrix conditioning within the regression; if the range is small, with $\alpha \approx \beta$, or if both $\alpha$ and $\beta$ are low (less than $50 \mathrm{~ms}$ for models such as these), then the RIEC algorithm would become much more susceptible to bias from equation errors.

\section{PARAMETER ESTIMATION AND MODEL VALIDATION}

The first regression, to estimate tyre stiffness from equations (23a) and (24a) is relatively trivial, as $x_{1}$ and $F_{\mathrm{t}}$ are linearly related for 99 per cent of the test duration; both algorithms estimate $K_{\mathrm{t}}$ with a very slight bias. Mass estimation represents a more challenging identification problem, however; Table 3 summarizes the results from

Table 3 Identified masses and tyre stiffnesses

\begin{tabular}{lccc}
\hline & \multicolumn{3}{c}{ Value for the following } \\
\cline { 2 - 4 } $\begin{array}{l}\text { Parameter } \\
\text { units })\end{array}$ & RIEC & DOLS & Source model \\
\hline$K_{\mathrm{t}}(\mathrm{N} / \mathrm{mm})$ & 177.8 & 177.9 & 180 \\
$M_{\mathrm{s}}(\mathrm{kg})$ & 302.7 & 240.1 & 300 \\
$M_{\text {us }}(\mathrm{kg})$ & 23.3 & 24.0 & 30 \\
\hline
\end{tabular}

(a) RIEC

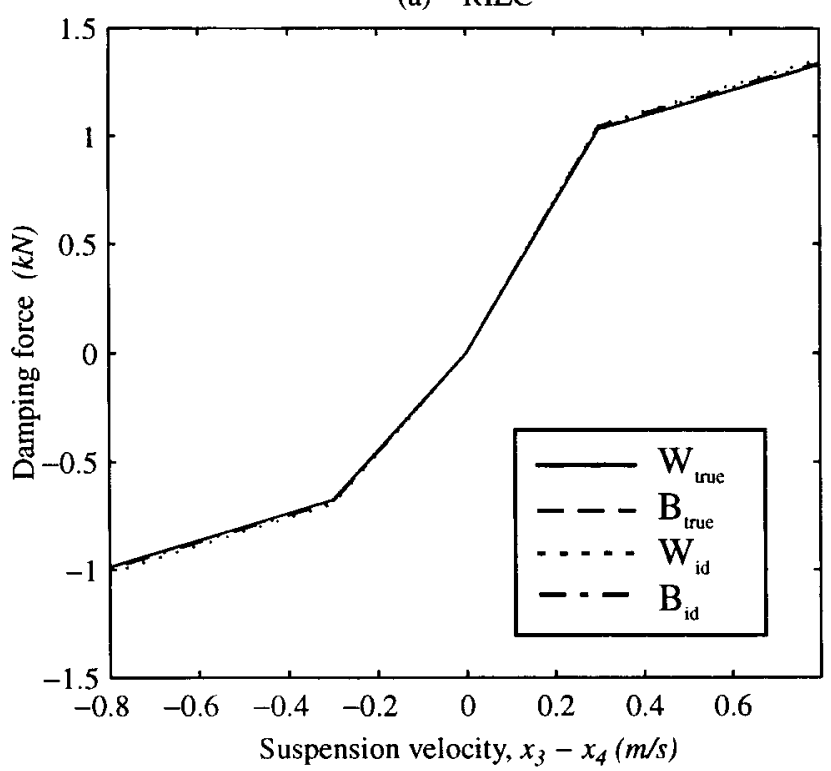

the regression equations (23a), (23b), (24a) and (24b). The engine mass disturbance has clearly affected the ability of DOLS to identify the total sprung mass, whereas the RIEC returns a satisfactory estimate. Both algorithms underestimate $M_{\text {us }}$ however, by about 20 per cent. Given the significant difference between the structures of the source and the identification models, the bias here may ultimately lead to more accurate identification models. However, as it is generally possible to estimate masses more accurately from engineering data, or simply by weighing, the case when the masses are known in advance will also be considered.

Four configurations are now available to complete the identification; the suspension force parameters can be identified from the motion of the wheel (W) or body (B) [regression equations (23c) and (24c) and equations (23d) and (24d) respectively] and, as both alternatives also require knowledge of one of the masses, this can be identified (subscript id) or known a priori (subscript true). It is interesting to consider all four variants, and these are denoted in the corresponding way, as $\mathrm{W}_{\text {true }}$, $\mathrm{B}_{\text {true }}, \mathrm{W}_{\mathrm{id}}$ and $\mathrm{B}_{\mathrm{id}}$. The resulting damper force values are illustrated in Fig. 7, and the spring stiffness estimates are given in Table 4; for reference, recall that the first-stage spring stiffness in the source model is $20 \mathrm{kN} / \mathrm{m}$.

Table 4 Identified suspension stiffness values

\begin{tabular}{llc}
\hline & \multicolumn{2}{c}{$K_{\mathrm{s}}(\mathrm{kN} / \mathrm{m})$} \\
\cline { 2 - 3 } Configuration variant & RIEC & DOLS \\
\hline $\mathrm{W}_{\text {true }}$ & 20.9 & 23.1 \\
$\mathrm{~B}_{\text {true }}$ & 20.7 & 19.7 \\
$\mathrm{~W}_{\text {id }}$ & 20.8 & 20.1 \\
$\mathrm{~B}_{\text {id }}$ & 20.9 & 15.8 \\
\hline
\end{tabular}

(b) DOLS

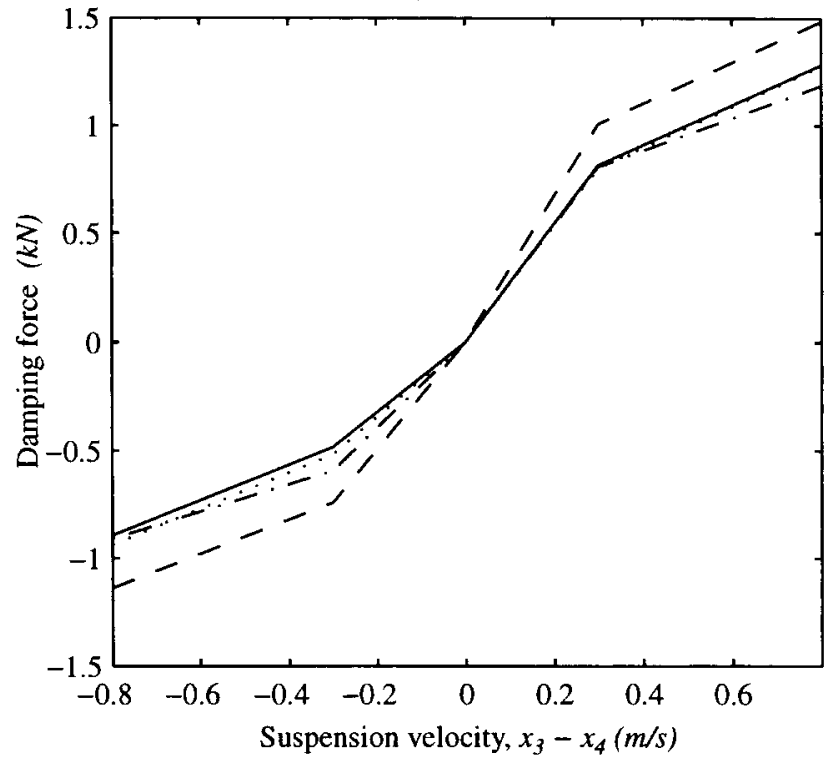

Fig. 7 Identified damper map 
The most striking observation here is the consistency of the RIEC estimations, for $F_{i}$ and $K_{\mathrm{s}}$. Conversely, the DOLS results vary considerably. We might expect a biased DOLS result from $B_{\text {id }}$ (which uses $M_{\mathrm{s}}=240.1$ ) and this is seen in a very low estimate of $K_{\mathrm{s}}$. However, DOLS does not return a consistent result even when known masses are employed; note particularly the extreme damper map, in the $\mathrm{B}_{\text {true }}$ variant.

As suggested earlier, the validity of these identification models cannot be assessed simply in terms of parameter matches with the source data; the source and identification models are too dissimilar. Validation simulations are thus carried out, to compare state trajectories from source and identification models. However, the road input must be carefully chosen for these; although it is reasonable to use broad-band excitation to ensure good conditioning in the identification process, the validation should reflect more general conditions for which the model might be required.

To this end, two new input sequences are introduced. The first represents random road roughness, a Gaussian white noise process coloured using a filter of the form proposed by Robson [6]. The vertical displacement power spectral density (PSD) $S$ of the surface is modelled as a function of frequency $f$ by

$$
S(f)=k U^{1.5} f^{-2.5}
$$

where the vehicle speed $U$ is chosen to be $20 \mathrm{~m} / \mathrm{s}$ and the roughness coefficient $k$ is $3 \times 10^{-5}$ to emulate a poorquality minor road.

The second input considers a single event, a nominal pot-hole the profile for which is given in Fig. 8; again this is traversed at $20 \mathrm{~m} / \mathrm{s}$. Figures 9 and 10 show validation results for both the DOLS and the RIEC models identified in the $B_{\text {id }}$ variant. In each case, the four common state variables $x_{1}$ to $x_{4}$ are plotted. To provide a quantitative measure of performance, the percentage error $p$ is defined for the $i$ th state as

$$
p_{i}=\frac{\sqrt{ }\left\{\sum_{k}\left[\hat{x}_{i}(k T)-x_{i}(k T)\right]^{2}\right\}}{\sqrt{ }\left[\sum_{k} x_{i}^{2}(k T)\right]} \times 100
$$

where $\hat{x}$ and $x$ refer to the identification and the source data respectively.

The Robson road validation in Fig. 9 shows that both identification models provide a generally accurate reconstruction of the source data. For the DOLS result, a

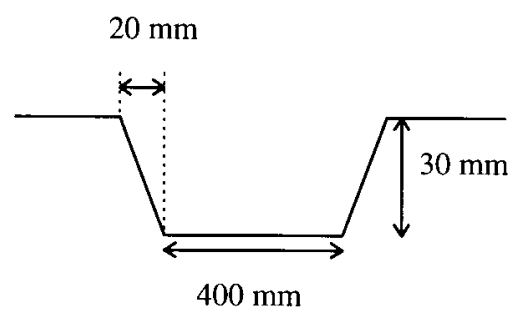

Fig. 8 Impulse event road profile poor parameter match does not necessarily result in poor model performance; in this case, low values for $K_{\mathrm{s}}$ and $M_{\mathrm{s}}$ combine to estimate the body-bounce mode well, although noticeable errors remain at low frequencies, particularly in $x_{1}$.

In Fig. 10, the pot-hole input provides a relatively severe double impulse, making the tyre lift; this is reflected in high errors in estimation of states $x_{1}, x_{2}$ and $x_{3}$ for both models. RIEC provides a slightly more accurate model, most notably in the first overshoot of $x_{4}$.

A summary of validation performance on each input condition is given in Table 5. The figures show aggregate percentage error (on the simple assumption that the accuracies in each of the four states have equal importance). In all variants the RIEC provides lower validation errors than the DOLS method, particularly for the Robson road input condition, where errors are reduced by about a third in most cases. The notable exception is the $\mathrm{B}_{\text {true }}$ DOLS model which returns an uncharacteristically good validation on the pot-hole input. Surprisingly, the most successful models on the Robson road are those which have the least accurate parameters. For the DOLS method, the $\mathrm{B}_{\mathrm{id}}$ model (illustrated in Fig. 9) has severely biased $M_{\mathrm{s}}$ and $K_{\mathrm{s}}$ estimates and, for both identification methods, imposition of the true masses results in less successful models. Although this last trend is reversed for the pot-hole validations, it may be concluded that the most 'accurate' parameters are not necessarily those which provide the best (simplified) model for the identified system.

\section{FURTHER STUDY OF VARIATIONS}

On the basis of the initial study above, the RIEC offers an advantage over the DOLS model in terms of accuracy, and appears to be more robust to simple changes in the identification process. Now the study is extended to examine robustness to changes in the underlying random processes. Two factors are considered: the resonance frequency of the principal disturbance mode, and choice of the input process bandwidth, which affects the degree to which all modes are excited.

The source model of Fig. $4 \mathrm{a}$ can easily be modified to move the engine vibration frequency and hence to emulate different sources of disturbance. Varying the mount stiffness $K_{\mathrm{m}}$ of the linearized model, and altering $B_{\mathrm{m}}$ to maintain a constant damping ratio, two cases are considered in addition to the original 'pathological' case. The first places the disturbance frequency at around $6 \mathrm{~Hz}$, between the wheel-hop and body-bounce modes; the second emulates the more general physical case, where the unmodelled disturbance exists at relatively high frequencies; resonance in this case occurs at $25 \mathrm{~Hz}$.

In the linearized form the three cases are summarized in Table 6. For each case, the non-linear mount characteristic is reconstructed in the source model simply by 

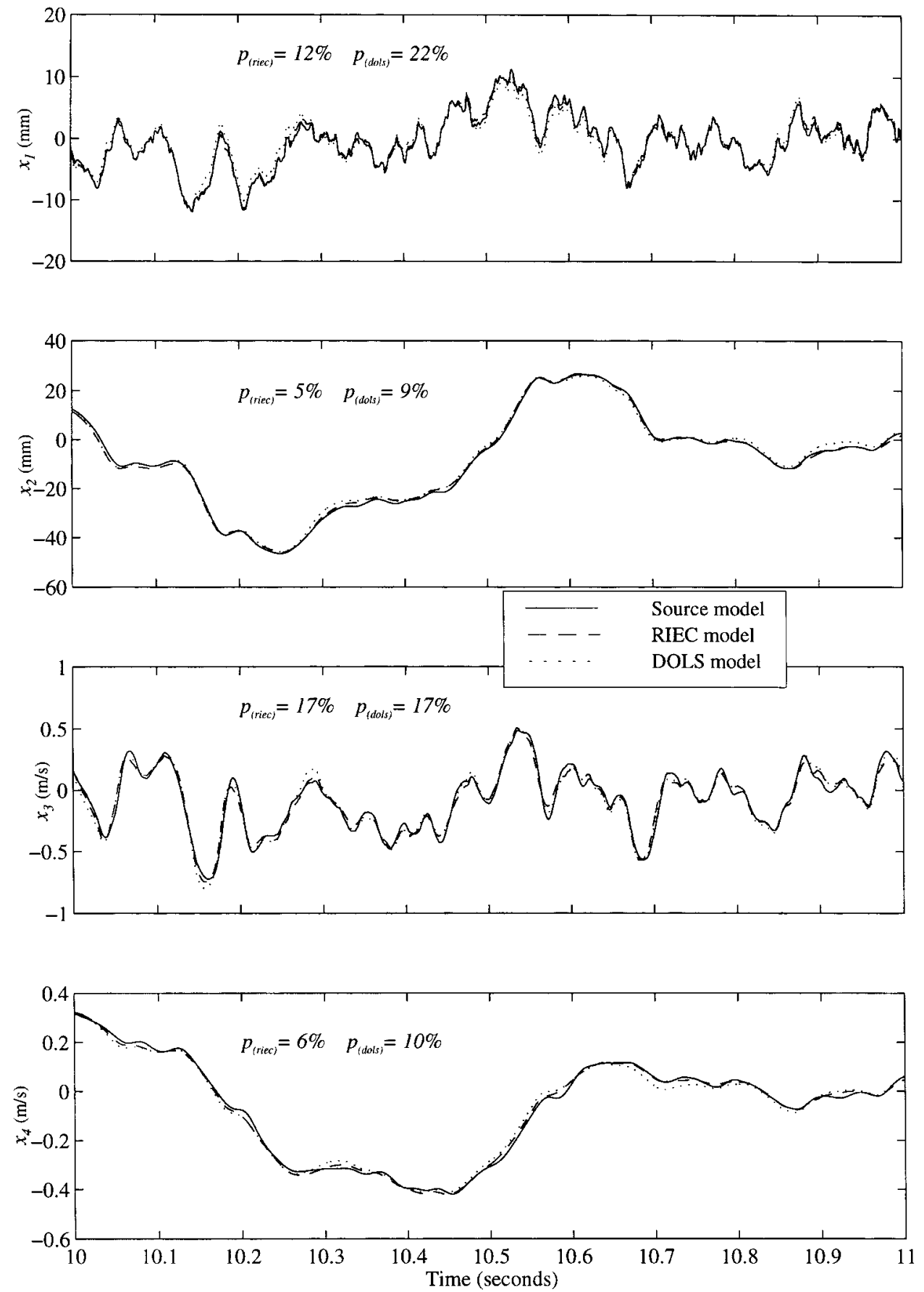

Fig. $9 B_{\text {id }}$ model validation on the 'Robson' road

applying the appropriate gain factor to the original spline model of Fig. 5c.

For the second factor, the original input signal $v_{\mathrm{r}}$ of Table 2 is filtered using the fast Fourier transform, to remove all frequencies above $f_{\max }$; source data are then generated and the parameters identified, for values of $f_{\max }$ between 0.5 and $30 \mathrm{~Hz}$. This was carried out for each of the three source models in the same way as in Section 4, except that here only the most successful identification is considered, via the equation of motion of the body mass, and using identified values for the two mass parameters ( $B_{i d}$ variant).

As before, the first-stage estimation of $K_{\mathrm{t}}$ remains relatively trivial for all input bandwidths; these results are not illustrated. Identification of the masses, and the subsequent estimation of $K_{\mathrm{s}}$ is presented in Fig. 11. The most significant feature of these plots is that the RIEC results are generally much less sensitive to the input bandwidth. The pathological and included-mode cases exhibit similar trends, although the latter generates 

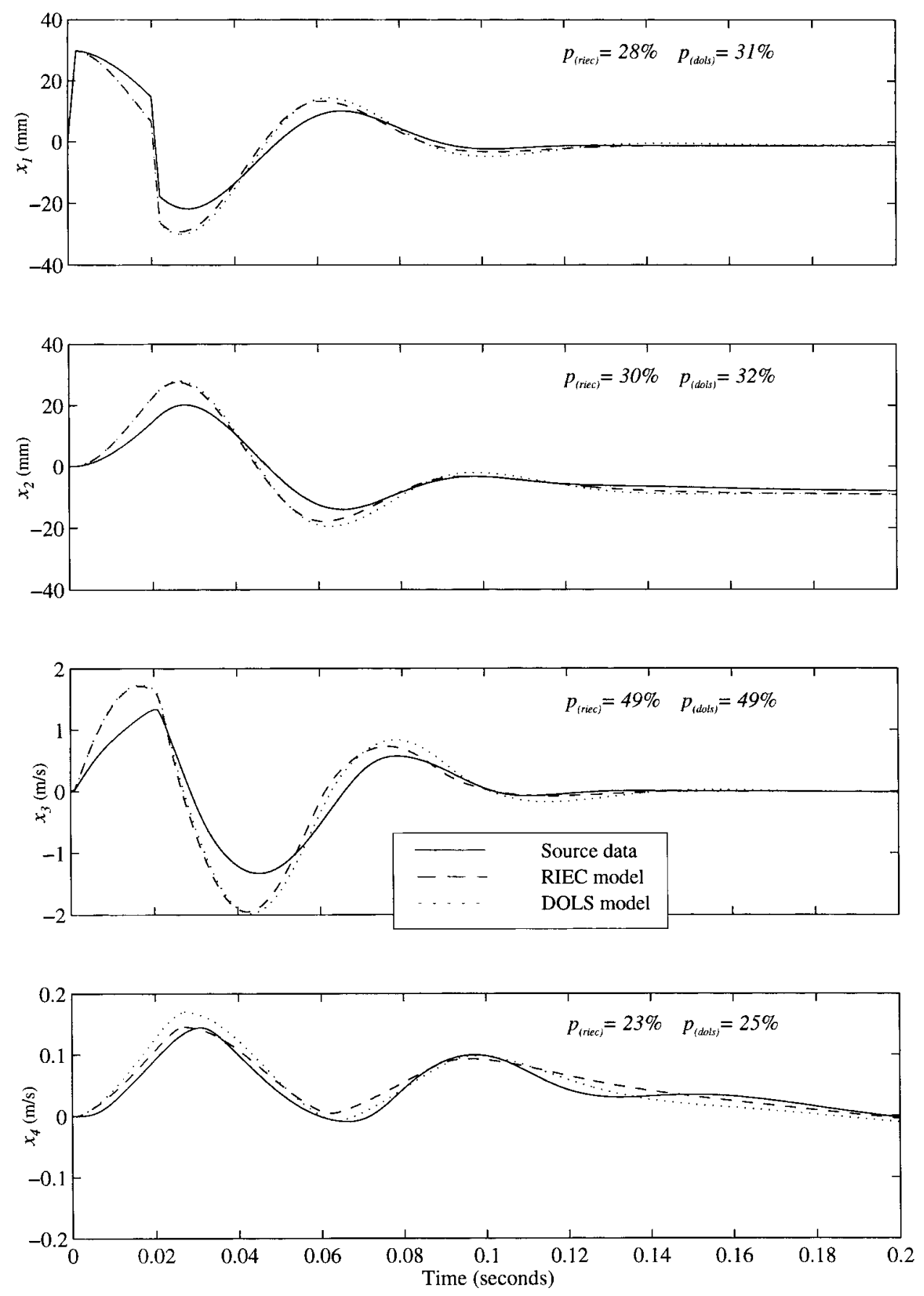

Fig. $10 B_{\text {id }}$ model validation for the pot-hole input

Table 5 The aggregate validation performance

\begin{tabular}{llllll}
\hline & \multicolumn{4}{c}{$\sum_{i} p_{i}$} \\
\cline { 2 - 3 } $\begin{array}{l}\text { Configuration } \\
\text { variant }\end{array}$ & 'Road' input condition & & \multicolumn{2}{c}{ 'Pot-hole' input condition } \\
\cline { 2 - 3 } \cline { 5 - 6 } \cline { 5 - 6 } & RIEC & DOLS & & RIEC & DOLS \\
\hline $\mathrm{W}_{\mathrm{id}}$ & 41.0 & 70.0 & & 126.2 & 138.8 \\
$\mathrm{~B}_{\mathrm{id}}$ & 40.2 & 57.5 & & 129.8 & 137.7 \\
$\mathrm{~W}_{\text {true }}$ & 58.9 & 87.6 & & 116.2 & 127.8 \\
$\mathrm{~B}_{\text {true }}$ & 59.4 & 65.9 & & 116.9 & 95.8 \\
\hline
\end{tabular}

Table 6 The parameters defining the variants of the source model

\begin{tabular}{lccc}
\hline & $\begin{array}{l}\text { Engine resonance } \\
\text { frequency }(\mathrm{Hz})\end{array}$ & $K_{\mathrm{m}}(\mathrm{kN} / \mathrm{m})$ & $B_{\mathrm{m}}(\mathrm{N} \mathrm{s} / \mathrm{m})$ \\
\hline Pathological case & 13 & 400 & 4000 \\
Included-mode case & 6 & 100 & 2000 \\
Higher mode case & 25 & 1500 & 7750 \\
\hline
\end{tabular}



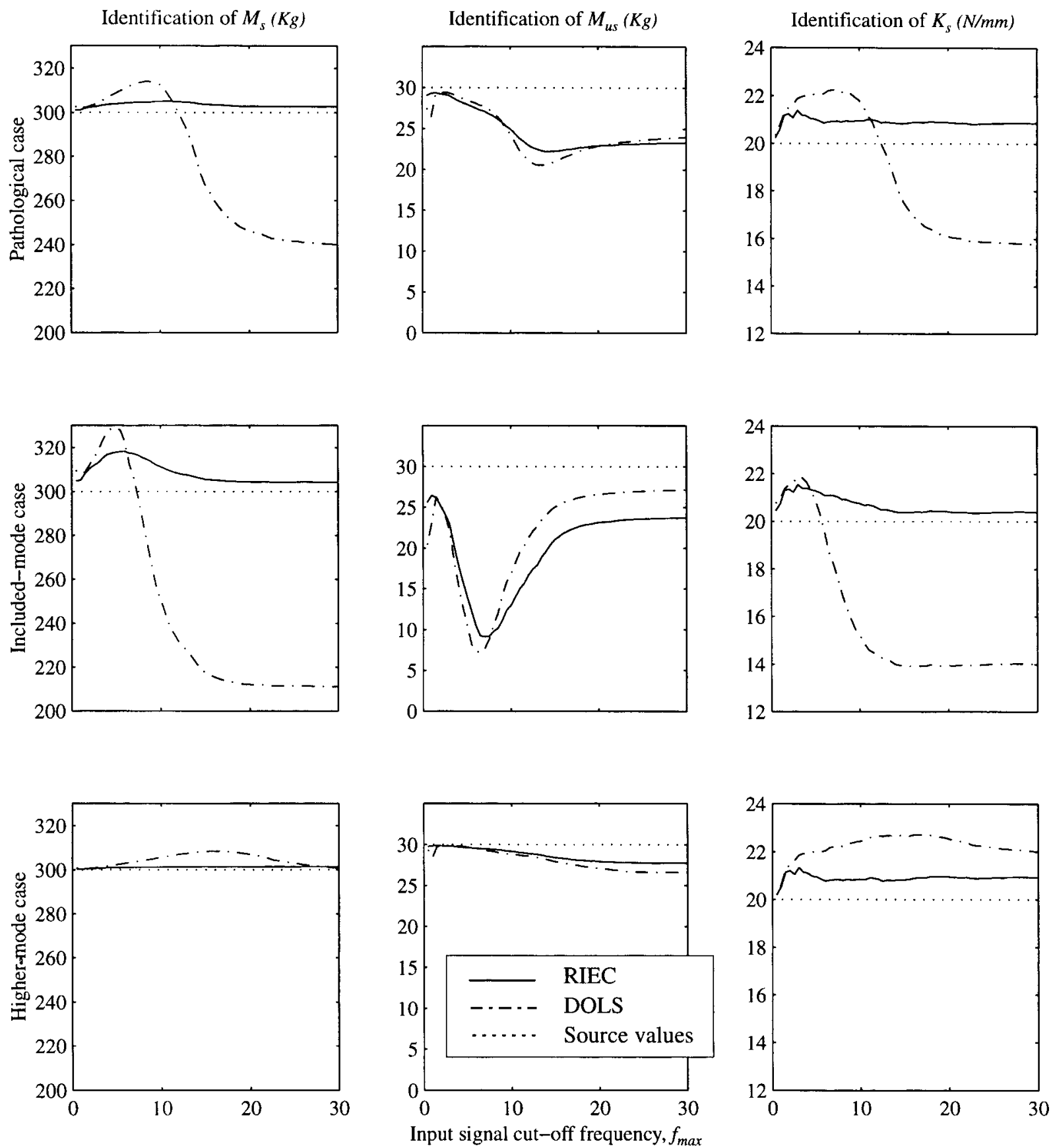

Fig. 11 Parameters identified as the input bandwidth is varied

greater parameter variation with respect to the bandwidth and, for the DOLS model, a more severe bias occurs in $M_{\mathrm{s}}$ and $K_{\mathrm{s}}$. Interestingly, when the bandwidth includes the wheel-hop mode (above about $15 \mathrm{~Hz}$ ), the RIEC identification in both of these cases is very similar. Indeed there is little difference between the RIEC models identified at higher bandwidths in all three cases. The higher mode creates less parametric variation for both methods, although again the RIEC curves provide a more consistent set of results.

Figure 12 illustrates variations in the identified damper map. Rather surprisingly, the results are very similar in all three cases; therefore only the pathological case is illustrated here. Again the RIEC shows a consistent identification, with almost constant $F_{i}$ after about $10 \mathrm{~Hz}$, whereas the DOLS method exhibits a drift in all four forces, towards lower values as the input frequency increases.

Consideration of equation errors shows that the damper compliance has a significant effect here. Figure 13 shows two identified damper maps together with the corresponding scatter plots of the damper force versus the suspension velocity $x_{3}-x_{4}$ from the source data. In Fig. 13a the input bandwidth is low and the 

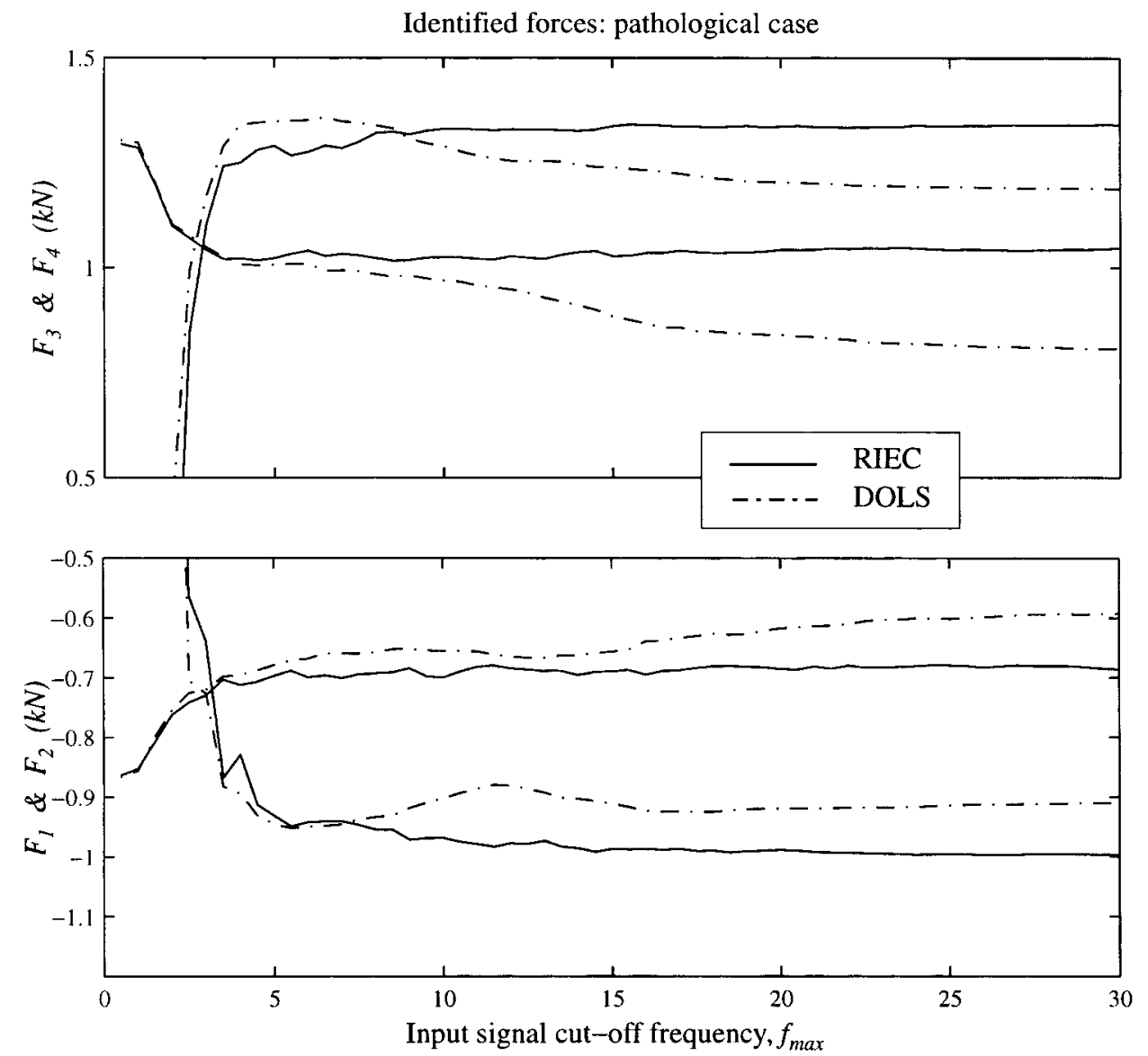

Fig. 12 Identified damper forces over varying input bandwidth

(a) $f_{\max }=5 \mathrm{~Hz}$

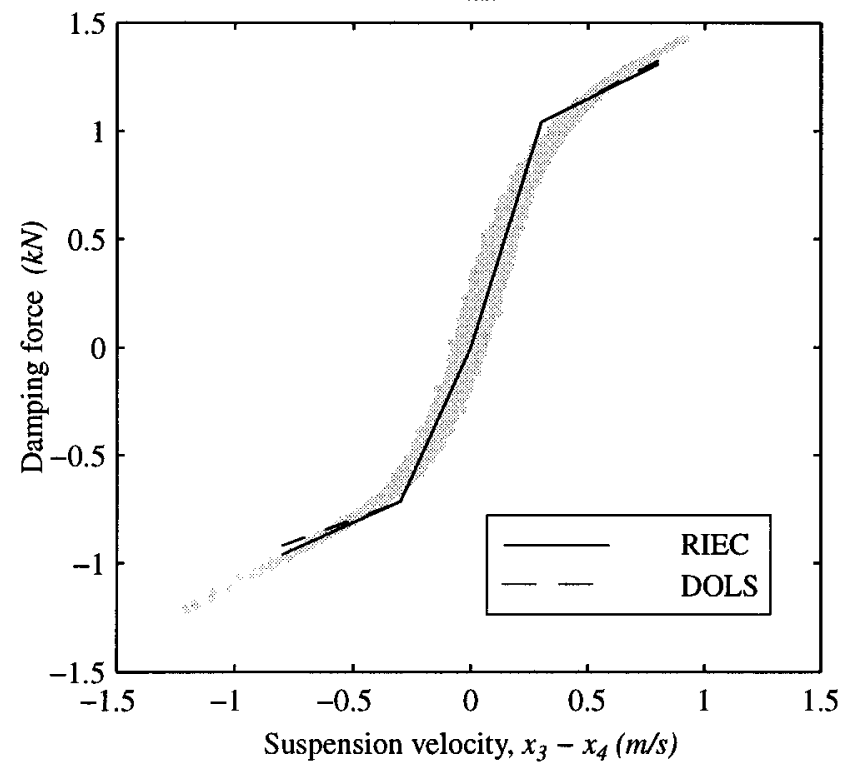

(b) $f_{\max }=30 \mathrm{~Hz}$

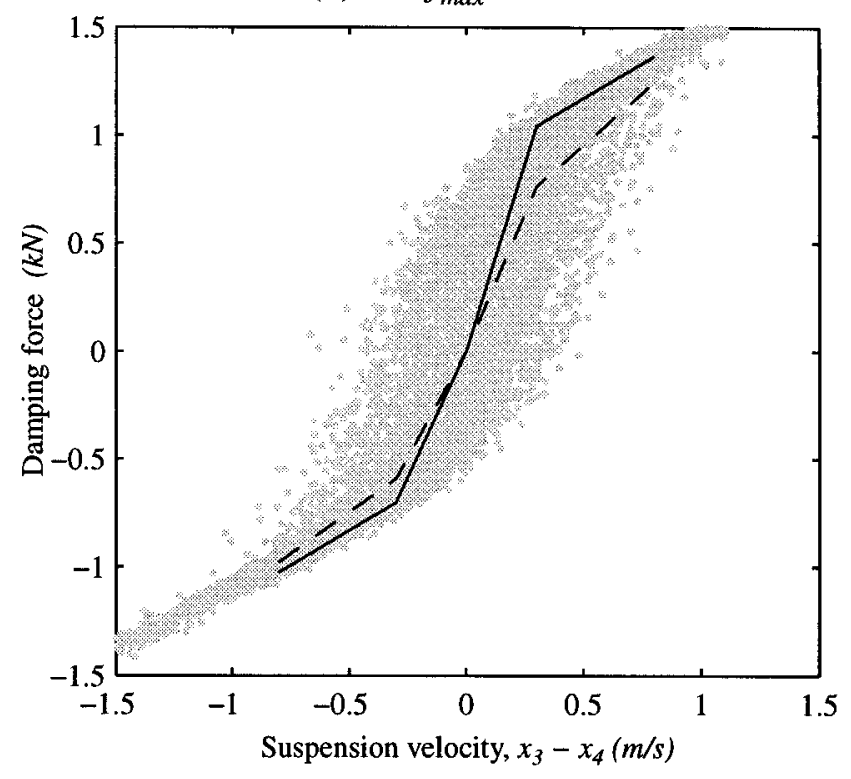

Fig. 13 Comparison of damper identifications at different input bandwidths

compliant element induces only slight hysteresis. As the bandwidth increases to excite the wheel-hop and the engine modes in Fig. 13b, however, the effect becomes significant. Although the damper force is not directly available within the regression, it is clear that the parameter drift in Fig. 12 is explained by this hysteresis, with the DOLS damper map shifting to adopt the line of least instantaneous force error. For the RIEC, the identified 
$F_{i}$ remain much more consistent, perhaps because the method considers an aggregate of force error, over time periods typically longer than the wheel-hop and engine mode cycles. Over each complete cycle, the net impulse exerted by the compliant element is approximately zero; thus the RIEC rejects these bounded errors in the same way as those due to the unmodelled engine mode.

To complete the analysis, the identification models are again validated using the two inputs described in Section 4. Again the aggregate performance for the four states is considered, and Fig. 14 shows how this varies with the bandwidth $f_{\max }$. These plots effectively summar-

Robson road (Road 1)
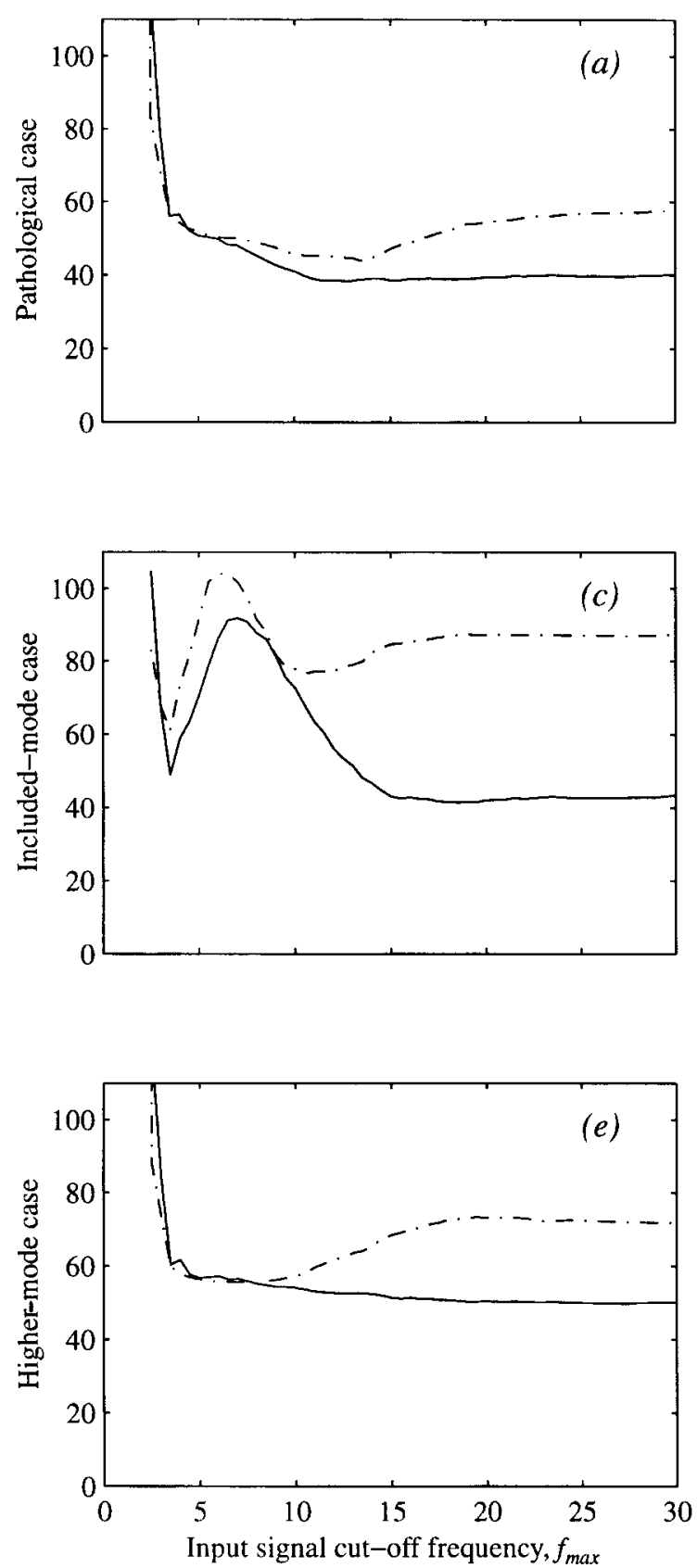

ize the whole study and they reveal a number of interesting points.

Firstly, the RIEC models show a consistent trend of diminishing $p$ as $f_{\max }$ increases; in all except one plot the highest bandwidth input provides the most accurate model. This is an intuitively correct result, and critically it is not the case for the DOLS data; in all cases on the Robson road, better results can be obtained by selective excitation of frequencies.

For the pathological and included-mode cases, the performance plots do not display the oscillatory variations of $M_{\mathrm{s}}$ and $K_{\mathrm{s}}$ seen at low bandwidths for the DOLS
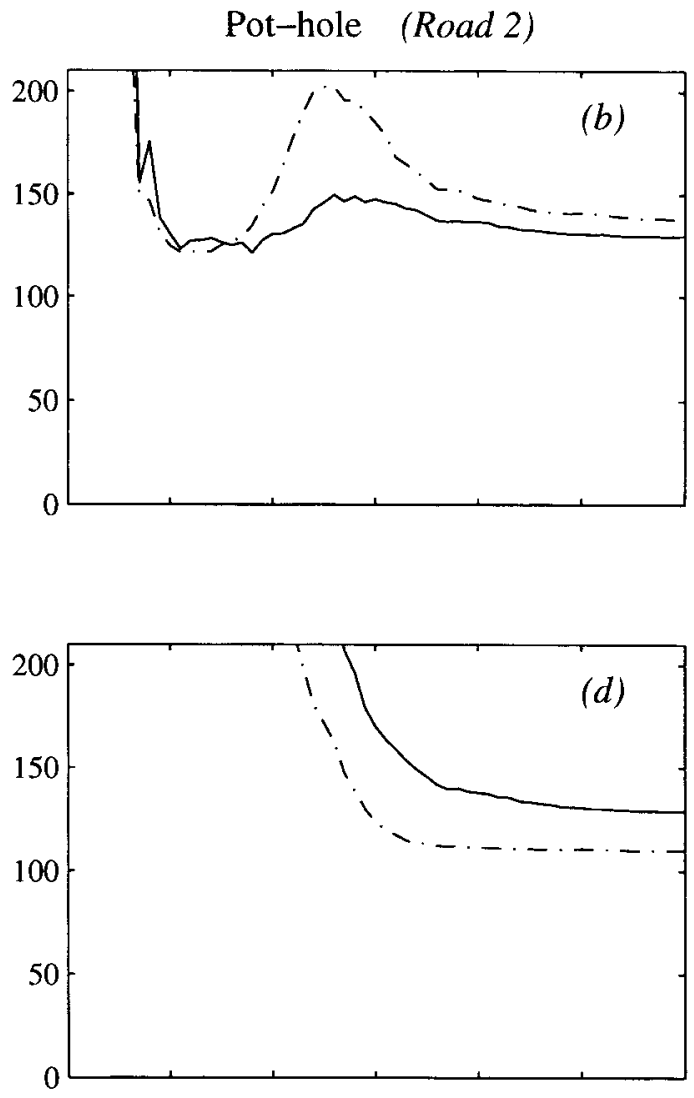

Fig. 14 Aggregate validation performance $(p)$ as the identification input bandwidth varies 
model in Fig. 11. As with the initial study this shows how the relatively ill-conditioned parameters $M_{\mathrm{s}}$ and $K_{\mathrm{s}}$ can vary greatly while inducing relatively low model error.

Conversely, the peaks in Figs $14 \mathrm{~b}$ and c show good correlation with the parameter bias of Fig. 11; variations in $M_{\text {us }}$ match the performance trends for both the RIEC and the DOLS models. Further investigation shows that the errors in the wheel velocity $p_{3}$ predominate in these validations, and the obvious inference is that better models can be obtained with more accurate estimation of unsprung mass. However, the results for true masses in Table 5 refute this. Also there is a clear difference in sensitivity to the bias in $M_{\text {us }}$ between the DOLS and RIEC models in Fig. 14b.

A final point of interest from these plots is the large increase in $p$ for DOLS over the higher-bandwidth tests in Figs 14e and f; this occurs in spite of relatively consistent values for the identified parameters, shown for the higher-mode case in Fig. 11. The error trend correlates with variations in $M_{\text {us }}$ and the damper forces, but similar variations in the first two cases result in less severe changes in performance. It appears again that the DOLS model is simply more sensitive to parameter variations for this higher-mode case.

\section{CONCLUSIONS}

On the basis of the simulation studies carried out in this paper, use of the new RIEC represents an attractive basis for identification of reduced order models. The method is successful in rejecting errors in the source data arising from unmodelled modes.

In all cases the new algorithm results in more accurate identification models than those produced by the reference DOLS method. However, the most striking result is the degree of consistency with which parameters are identified under the RIEC; essentially the same model is derived from four permutations of the dynamic equations of motion, and in the presence of three very different unmodelled modes. In most cases this model is also well retained under variation in the input bandwidth.

It should also be noted that the RIEC method successfully identifies appropriate mass parameters where the reference method fails. This is particularly significant given the finding that the 'true' source model masses provide suboptimal parameter choices for the identification models examined.

The new method is simple, requiring only minor preprocessing in the generation of the regressor set and retaining a straightforward least-squares optimization. There are also additional advantages, e.g. in the ability to extract a large number of samples from each single batch of data, and in the ability to tune the integration step length depending on the system to be modelled. Further research, using physical examples, will provide an important platform for development of the scheme, particularly in terms of the relative importance of measurement errors, not considered in this study.

\section{REFERENCES}

1 Norton, J. P. An Introduction to Identification, 1986 (Academic Press, London).

2 Söderström, T. and Stoica, P. G. System Identification, 1989 (Prentice-Hall, New York)

3 Press, W. H., Teukolsky, S. A., Vetterling, W. T. and Flannery, B. P. Numerical Recipes: The Art of Scientific Computing, 1988 (Cambridge University Press, Cambridge).

4 Best, M. C. On the modelling requirements for the practical implementation of advanced vehicle suspension control. PhD thesis, Loughborough University, 1995.

5 Lin, Y. and Kortüm, W. Identification of system physical parameters of vehicle systems with nonlinear components. Veh. System Dynamics, 1991, 20, 354-365.

6 Robson, J. D. Road surface description and vehicle response. Int. J. Veh. Des., 1979, 1, 25-35. 